ARTIGO ORIGINAL ORIGINAL ARTICLE

\title{
Custo direto da judicialização dos sistemas de infusão contínua de insulina (Sici) no município do Rio de Janeiro
}

\author{
Direct cost of the judicialization of continuous insulin \\ infusion systems (Sici) in the city of Rio de Janeiro
}

Viviane Silva Telheiro', Bruna Medeiros Gonçalves de Veras², Luciana Tarbes Mattana Saturnino³

DOI: 10.21115/JBES.v12.n1.p71-80

Palavras-chave:

judicialização da saúde, sistemas de infusão de insulina, custo

\section{Keywords:}

health's judicialization, insulin infusion systems, cost

\section{RESUMO}

Objetivo: Estimar o custo direto anual da judicialização dos sistemas de infusão contínua de insulina (Sici), no município do Rio de Janeiro, entre 2015 e 2017, e analisar as características dos processos relacionados à referida tecnologia. Métodos: Estudo transversal descritivo que analisou os pareceres técnicos emitidos pelo Núcleo de Assessoria Técnica em Ações de Saúde e os respectivos processos judiciais. As informações relacionadas aos custos foram provenientes do relatório de solicitação de empenho da Central de Atendimento às Demandas Judiciais do Estado do Rio de Janeiro e de orçamentos fornecidos pelos fabricantes/distribuidores. Resultados: Foram avaliados 189 processos emitidos. A média de idade dos autores dos processos foi de 25,1 anos e $57,1 \%$ eram do sexo feminino. Em 98 casos (51,9\%), o documento utilizado no processo foi proveniente de médicos da rede pública de saúde. O juiz deferiu o pleito em 153 processos (80,9\%). De um total de 75 médicos prescritores, nove foram responsáveis por $43,9 \%$ das prescrições, demonstrando uma concentração das prescrições em determinados profissionais. O valor estimado de custo direto acumulado (20152017) decorrente da judicialização dos Sici, no município do Rio de Janeiro, corresponde a $R \$ 6,9$ milhões, o que representa 6,7\% dos gastos de judicialização da saúde no mesmo período. Conclusão: Considerando que a tecnologia analisada é recorrentemente judicializada, os dados coletados podem gerar informações úteis para os gestores governamentais da área da saúde, na avaliação da eficiência na alocação dos recursos públicos, para os profissionais que trabalham na assistência judiciária e para os juízes.

\begin{abstract}
Objective: To estimate the annual direct cost of the Judicialization of Continuous Insulin Infusion Systems (Sici), in the city of Rio de Janeiro, between 2015 and 2017. Methods: It is a cross sectional study that analyzed the technical opinions on the Sici issued by the Core of Technical Advice on Health Actions and the respective judicial processes. The information related to the costs was obtained from the Report of Request for Compromise provided by the Center of Attendance to the Lawsuits of the state of Rio de Janeiro and budgets of the manufacturer /distributors. Results: There were 189 processes and the average age of the authors was 25.1 years and that the majority was female (57.1\%). In 98 cases (51.9\%) the document used in the process came from doctors of the public health network. The judge granted the suit in 153 cases (80.9\%). Nine physicians were responsible for $43.9 \%$ of the prescriptions, out of a total of 75 prescribing physicians, demonstrating a concentration of prescribing physicians. The estimated value of accumulated direct cost (2015-2017), due to the Sici's judicialization in the city of Rio de Janeiro, corresponds to $R \$ 6.9$ million, which represents $6.7 \%$ of health care expenditures in the same period. Conclusion: Considering that the technology
\end{abstract}

Recebido em: 20/01/2020. Aprovado para publicação em: 09/04/2020.

1. Mestre pelo Núcleo de Avaliação de Tecnologias em Saúde do Instituto Nacional de Cardiologia, Rio de Janeiro, RJ, Brasil.

2. Mestre em Engenharia Biomédica pela Universidade Federal do Rio de Janeiro (UFRJ), Rio de Janeiro RJ, Brasil.

3. Doutora em Ciências Farmacêuticas pela Faculdade de Farmácia da Universidade Federal de Minas Gerais (UFMG), Belo Horizonte, MG, Brasil.

Congressos: Parte do presente estudo foi apresentado no Primeiro Congresso da Rede Brasileira de Avaliação de Tecnologias em Saúde, realizado em Brasília - DF, em 1 a 4 de outubro de 2019.

Fontes de financiamento: Não houve financiamento para a elaboração deste estudo.

Conflitos de interesses: B. M. G. Veras e L. T. M. Saturnino são funcionárias da GSK Brasil e professoras do Mestrado em Avaliação de Tecnologia em Saúde do Instituto Nacional de Cardiologia (INC). Esse material se refere a uma produção técnica pelo INC e não há conflitos de interesses. V. S. Telheiro não apresenta conflitos de interesses.

Autor correspondente: Viviane Silva Telheiro. Avenida Rainha Elizabeth da Bélgica, 201, ap. 304, Copacabana, Rio de Janeiro, RJ, Brasil. CEP: 22081-031. 
under review is repeatedly judicialized, the data collected can generate useful information to public healthcare decision makers, the evaluation of efficiency in the allocation of public resources, and professionals working in legal aid or for the judges themselves.

\section{Introdução}

O primeiro registro de mandado judicial na Secretaria de Estado de Saúde do Rio de Janeiro data de 1991. No início, as ações envolvendo o direito à saúde estavam relacionadas a algumas enfermidades, principalmente à doença por vírus da imunodeficiência humana (HIV). Contudo, a partir do ano 2000, houve um grande aumento de ações judiciais contra o estado do Rio de Janeiro para a obtenção de vários tipos de medicamentos (Messeder et al., 2005).

Dada a importância do tema, o aumento da demanda judicial em saúde tem sido abordado recorrentemente nos estudos científicos (Figueiredo, 2010; Pierro, 2017). No entanto, grande parte desses estudos versa sobre medicamentos, pois esses são os itens mais judicializados, conforme destacam Oliveira et al. (2015).

Apesar da importância do estudo da judicialização de medicamentos, também é necessária a realização de pesquisas que identifiquem o impacto da judicialização de outras tecnologias. Nesse contexto, destaca-se o aumento da demanda por equipamentos e insumos relacionados à saúde, uma vez que há doenças, como é o caso do diabetes mellitus (DM) tipo 1, que requerem a utilização dessas tecnologias.

Embora existam medicamentos e insumos padronizados para o tratamento da DM, muitas pessoas com essa doença recorrem ao Poder Judiciário para a obtenção dos sistemas de infusão contínua de insulina (Sici), compostos pela bomba de infusão de insulina (BIC) e pelos insumos para a manutenção do tratamento com a bomba, que não são padronizados para a dispensação pelo Sistema Único de Saúde (SUS).

Nesse contexto, o objetivo primário deste estudo é apurar os custos diretos anuais dos Sici judicializados, no município do Rio de Janeiro, entre os anos de 2015 e 2017. Os objetivos secundários são: identificar a frequência de processos judiciais referentes a solicitações de Sici, analisar as características dos processos judiciais relacionados aos Sici e identificar os custos de cada item relacionado ao Sici que foi demandado.

\section{Métodos}

Trata-se de um estudo transversal descritivo com coleta secundária de dados contidos nos pareceres técnicos sobre os Sici, emitidos entre janeiro de 2013 e dezembro de 2017. A escolha quanto ao período de início da análise se deve ao Ato Normativo TJ no 05/2012, emitido em $1^{\circ}$ de fevereiro de 2012, que determinou que os processos envolvendo solicitação de itens relacionados à saúde fossem encaminhados ao Núcleo de Assessoria Técnica em Ações de Saúde do Tribunal de Justiça do Estado do Rio de Janeiro (NAT/TJ).
Para a identificação de pareceres técnicos e processos judiciais associados ao Sici, foram considerados os pareceres emitidos para processos que tinham como pleito os Sici ajuizados por autores residentes no município do Rio de Janeiro, sendo excluídos da amostra aqueles em duplicidade, ou seja, emitidos para um mesmo autor.

A coleta dos dados ocorreu somente após a obtenção de autorização do NAT para o uso de dados e aprovação do Comitê de Ética em Pesquisa (CEP) do Instituto Nacional de Cardiologia, sob o número 81035417.1.0000.5272.

Os dados coletados nos pareceres técnicos e nos processos sobre os Sici (Quadro 1) foram utilizadas com o intuito de traçar o panorama geral da judicialização dos Sici no município do Rio de Janeiro, bem como para identificar a representatividade dos pareceres relativos aos Sici, no total de pareceres emitidos pelo NAT.

Para a identificação dos custos diretos associados aos processos judiciais relativos ao Sici, foi feita uma tabulação descritiva dos dados dos pareceres técnicos do NAT/TJ e do NAT em Ações de Saúde da Justiça Federal (NAT/JF). Esses núcleos são responsáveis pela emissão de pareceres técnicos, com o intuito de subsidiar a tomada de decisão do juiz no julgamento das ações relacionadas à saúde.

Foram utilizadas várias fontes de informação de custos para o cálculo do impacto dessa judicialização no sistema, dentre elas, vale destacar, o Portal de Transparência do Município do Rio de Janeiro, o Portal de Transparência do Estado do Rio de Janeiro, a Central de Atendimento de Demandas Judiciais (CADJ) da Secretaria Estadual de Saúde (SES) e a Gerência de Atendimento a Mandados Judiciais da Secretaria Municipal de Saúde (SMS). Posteriormente, foi realizada a análise dos custos diretos da bomba de insulina e dos respectivos insumos dos processos identificados nos anos de 2015 a 2017 no município do Rio de Janeiro. Essa análise foi realizada para o período supracitado de modo a garantir maior precisão em sua apuração.

Os itens utilizados para analisar o custo direto dos Sici incluíram: a bomba de insulina das marcas Accu-Chek e Medtronic, os insumos para o tratamento com o Sici e insumos para a monitorização contínua de glicose intersticial (Quadro 2).

Para o volume judicializado, considerou-se o número de processos deferidos (analisados pelo NAT/TJ e NAT/JF). Com relação aos insumos necessários para o tratamento anual, foram consideradas as quantidades indicadas nos manuais dos fabricantes, no Guia Rápido de Manuseio Accu-Chek Combo, nas Diretrizes da Sociedade Brasileira de Diabetes (SBD) 2017-2018 ou nos orçamentos fornecidos pela Medtronic, contidos nos processos analisados. 
Quadro 1. Dados coletados nos processos de Sici

\begin{tabular}{|c|c|c|}
\hline Natureza do dado & Variável coletada & Descrição \\
\hline \multirow{4}{*}{$\begin{array}{l}\text { Dados } \\
\text { dos } \\
\text { pacientes }\end{array}$} & Sexo & Feminino/masculino \\
\hline & Idade & Data de nascimento \\
\hline & Hipossuficiência ou gratuidade de justiça & Sim/Não \\
\hline & Bairro de origem & Bairro de residência \\
\hline \multirow{9}{*}{$\begin{array}{l}\text { Dados relacionados } \\
\text { à saúde }\end{array}$} & Gravidez & Sim/Não \\
\hline & Origem do médico & Rede pública ou rede particular de saúde \\
\hline & Doença & Tipo de diabetes \\
\hline & Teste com Sici & Sim/Não \\
\hline & Marca prescrita & Identificação da marca \\
\hline & Marca pleiteada & Identificação da marca (Accu-Chek/Medtronic*) \\
\hline & Médico prescritor & $\begin{array}{l}\text { Identificação codificada do médico } \\
\text { prescritor (exemplos: A, B, C etc.) }\end{array}$ \\
\hline & Instituição de saúde & Identificação da instituição pelo nome \\
\hline & $\begin{array}{l}\text { Justificativa do médico para a prescrição } \\
\text { do tratamento com o Sici }\end{array}$ & Texto descritivo \\
\hline \multirow{5}{*}{ Dados do processo } & Representação legal & $\begin{array}{l}\text { Com representante (menor ou } \\
\text { incapaz) ou sem representante }\end{array}$ \\
\hline & Tipo de advogado & $\begin{array}{l}\text { Advogado público (defensoria pública) ou } \\
\text { advogado privado (advogado particular) }\end{array}$ \\
\hline & Identificação do advogado & $\begin{array}{l}\text { Identificação do advogado, cuja confidencialidade } \\
\text { será preservada por numeração (exemplos: } \\
\text { advogado 1, advogado } 2 \text { etc.) }\end{array}$ \\
\hline & $\begin{array}{l}\text { Existência de parecer da Câmara de } \\
\text { Resolução de Litígios em Saúde (CRLS) }\end{array}$ & Sim/Não \\
\hline & Decisão do juiz & Deferida/indeferida ou sem decisão \\
\hline
\end{tabular}

Fonte: Elaboração própria.

* Na descrição da marca, foi considerado Medtronic, apesar de se tratar do nome do fabricante, pois foi identificada, nos pareceres, a solicitação de três modelos diferentes do referido fabricante.

Quadro 2. Insumos e respectivos modelos considerados para a análise dos custos diretos dos Sici

\begin{tabular}{|c|c|}
\hline Bomba de insulina & Insumos para o tratamento com Sici \\
\hline \multirow[t]{4}{*}{ Accu-Chek (Flexlink) } & Conjunto de infusão \\
\hline & Aplicador de conjunto de infusão \\
\hline & Reservatório \\
\hline & Pacote de serviços \\
\hline \multirow[t]{8}{*}{ Medtronic } & Conjunto de infusão (MMT-396 ou 397) \\
\hline & $\begin{array}{l}\text { Aplicador de conjunto de } \\
\text { infusão (MMT-3951) }\end{array}$ \\
\hline & Reservatório (MMT-332A) \\
\hline & CareLink USB (MMT-7305NA) \\
\hline & $\begin{array}{l}\text { Insumos para a monitorização } \\
\text { contínua de glicose }\end{array}$ \\
\hline & Sensores de glicose (MMT-7708A) \\
\hline & Aplicador do sensor (MMT-7510) \\
\hline & Transmissor MiniLink (MMT-7707NA) \\
\hline
\end{tabular}

Para os valores e preços dos Sici, foram utilizadas as informações disponíveis nos orçamentos acostados em alguns processos. Como os orçamentos extraídos dos processos apresentavam valores distintos, em função de diferentes distribuidores ou do modelo do equipamento, considerou-se o orçamento mais conservador (com menor custo) para cada ano.

Os preços dos Sici da Medtronic relativos ao ano de 2015 indicados nos orçamentos são compatíveis com as informações de compra disponibilizadas pela CADJ/SES. Vale ressaltar que os modelos de bomba pleiteados mudaram ao longo do tempo, conforme o desenvolvimento do fabricante. Desse modo, a fim de viabilizar o cálculo dos custos, utilizou-se como modelo de referência da Medtronic o Paradigma ${ }^{\circ} \mathrm{Veo}^{\mathrm{TM}}$.

O custo relacionado aos insumos incorporados ao SUS e que também são utilizados no tratamento da DM tipo 1, mesmo nos casos dos pacientes que fazem uso do Sici, como tiras reagentes, glicosímetro, lancetas e medicamento (análogo de insulina), não foi incluído no cálculo do custo direto do Sici no município do Rio de Janeiro. 
O custo anual para cada insumo que não está padronizado pelo SUS foi obtido pela multiplicação da quantidade anual estimada pelo custo unitário apurado em cada ano.

\section{Resultados}

Entre os anos de 2013 e 2017 foi emitido um total de 21.861 pareceres, sendo 370 referentes a solicitações de Sici. O número total de pareceres emitidos em 2017 foi quase o dobro daqueles emitidos em 2013 ( $n=5.191$ e $n=2.789$, respectivamente). Nesse mesmo período, o percentual do total de pareceres que eram referentes aos Sici variou de 1\% (anos de 2015 e 2017) a $2 \%$ (demais anos). Na figura 1, são apresentados o total de pareceres no período e o percentual que se referiram aos Sici.

Para fins do cálculo do impacto dos custos da aquisição de emergência do Sici no orçamento da SES-RJ, foram considerados os anos de 2015 a 2017, o que corresponde a um total de 263 processos. Desses, 74 estavam duplicados, restando 189 para a análise. Do total analisado, 48,2\% possuíam representantes legais e 80,9\% foram ajuizados por meio da Defensoria Pública. A média de idade dos autores dos processos foi de 25,1 anos e 57,1\% eram do sexo feminino. Na maioria, os autores eram moradores da zona norte e da zona oeste do município do Rio de Janeiro, representando 44,9\% ( $n=85)$ e 39,2\% $(n=74)$, respectivamente (Tabela 1$)$.

Entre os 33 processos ajuizados por meio de advogados contratados pelos autores, 30,3\% $(n=10)$ corresponderam a um mesmo profissional. Vale destacar que $62,9 \%$ dos casos passaram previamente pela Câmara de Resolução de Litígios em Saúde, isto é, não houve sucesso na tentativa de solucionar a questão no âmbito administrativo. Quanto à decisão do juiz sobre o processo, observou-se que em $80,9 \%$ dos casos houve o deferimento dos itens pleiteados.

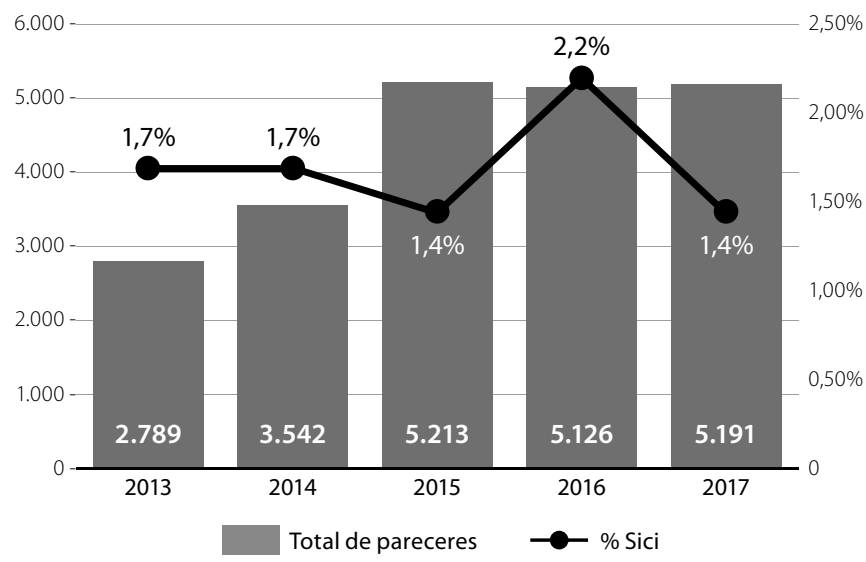

Fonte: Elaboração própria.

Nota: Foi considerado o total de pareceres emitidos pelo NAT, que englobam os pareceres emitidos pelo NAT/TJ e NAT/JF, entre 2013 e 2017.

Figura 1. Distribuição do total de pareceres emitidos e percentual referente aos Sici por ano (2013 a 2017).
Tabela 1. Características gerais dos processos de judicialização de Sici, no período de 2015 a 2017 ( $n=189)$

\begin{tabular}{|c|c|}
\hline Características dos autores & $\begin{array}{l}\text { Total de pareceres } \\
\qquad(\mathrm{n}=189)\end{array}$ \\
\hline Média de idade (intervalo) - anos & $25,1(0,6-65,8)$ \\
\hline Sexo feminino* n (\%) & $108(57,1)$ \\
\hline Possuía representante legal n (\%) & $91(48,1)$ \\
\hline Presença de hipossuficiência† n (\%) & $186(98,4)$ \\
\hline \multicolumn{2}{|l|}{ Zona de origem n (\%) } \\
\hline Norte & $85(45,0)$ \\
\hline Oeste & $74(39,2)$ \\
\hline Sul & $16(8,5)$ \\
\hline Centro & $13(6,9)$ \\
\hline Não identificada & $1(0,5)$ \\
\hline \multicolumn{2}{|l|}{ Características de saúde } \\
\hline Rede pública n (\%) & $98(51,9)$ \\
\hline \multicolumn{2}{|c|}{ Doença a qual se refere o processo $n(\%)$} \\
\hline DM1 & $182(96,3)$ \\
\hline DM2 & $4(2,1)$ \\
\hline LADA & $1(0,5)$ \\
\hline DM secundária à pancreactomia & $1(0,5)$ \\
\hline Não informado & $1(0,5)$ \\
\hline \multicolumn{2}{|l|}{ Uso anterior de Sici } \\
\hline Sim & $24(12,7)$ \\
\hline Apenas teste & $73(38,6)$ \\
\hline Não informado & $92(48,7)$ \\
\hline \multicolumn{2}{|l|}{ Marca prescrita/pleiteada n (\%) } \\
\hline Accu-Chek & $61(32,3) / 58(30,7)$ \\
\hline Medtronic & $117(61,9) / 118(62,4)$ \\
\hline Ambos & $1(0,5) / 2(1,1)$ \\
\hline Não descrita & $10(5,3) / 11(5,8)$ \\
\hline \multicolumn{2}{|l|}{ Características do processo } \\
\hline Defensoria pública & $153(81,0)$ \\
\hline Advogado particular & $33(17,5)$ \\
\hline Ambos & $3(1,6)$ \\
\hline Teve parecer CRLS & $119(63,0)$ \\
\hline Decisão deferida pelo juiz & $153(81,0)$ \\
\hline
\end{tabular}

Fonte: Elaboração própria. Dados extraídos dos processos judiciais e dos pareceres do NAT/TJ e NAT/JF, no período de 2015 a 2017.

Notas: * Apenas duas pacientes estavam grávidas e duas planejavam engravidar. † A gratuidade da justiça é aplicável aos casos de deferimento da solicitação de hipossuficiência.

DM1: diabetes mellitus tipo 1; DM2: diabetes mellitus tipo 2; LADA: diabetes autoimune latente do adulto; CRLS: Câmara de Resolução de Litígios em Saúde. 
Observou-se que $35,5 \%$ dos autores $(n=67)$ utilizaram a rede particular de saúde, no entanto recorreram à Defensoria Pública para ajuizar o processo. Por outro lado, observa-se que 10 autores (5,3\%) utilizaram a rede pública de saúde, mas ajuizaram o processo por meio de advogados privados.

Sobre o tratamento com o Sici, os documentos médicos dos processos analisados foram emitidos tanto por profissionais da rede pública de saúde $(51,9 \%)$ quanto da rede particular (48,1\%). Em relação aos prescritores, nove médicos foram responsáveis pela prescrição de $43,8 \%$ dos casos analisados, de um total de 75 médicos prescritores. Destaca-se que um dos médicos foi responsável por 19 prescrições, o que corresponde a $10,1 \%$ de todos os casos da amostra.

A Tabela 2 apresenta a distribuição das solicitações de Sici conforme as instituições públicas de origem dos documentos médicos utilizados nos processos. Nessa tabela, observa-se que 45,9\% foram provenientes do Instituto Estadual de Diabetes e Endocrinologia Luiz Capriglione (lede). Adicionalmente, 21 casos foram oriundos do Hospital Universitário Clementino Fraga Filho (HUCFF) e 13, do Instituto de Puericultura e Pediatria Martagão Gesteira (IPPMG).

Com relação aos casos de DM tipo $1(n=183)$, foram identificadas as seguintes comorbidades associadas à doença de base: cinco pacientes com retinopatia, quatro pacientes com retinopatia e neuropatia, três pacientes com retinopatia e nefropatia. Adicionalmente, cabe destacar que foi identificado um paciente para cada uma das seguintes comorbidades: re- tinopatia com doença cardiovascular, nefropatia, neuropatia e doença cardiovascular.

Destaca-se ainda que em 20 processos foram pleiteados somente os insumos para o Sici ou os insumos para monitorização contínua de glicose intersticial. Os motivos foram: a BIC havia sido comprada pelo autor da ação ou a BIC foi cedida

Tabela 2. Distribuição das solicitações conforme a instituição pública de origem $(n=98)$

\begin{tabular}{ll}
\hline Instituição pública & $\mathbf{n}(\%)$ \\
\hline lede & $45(45,9)$ \\
\hline HUCFF & $21(21,4)$ \\
\hline IPPMG & $13(13,3)$ \\
\hline Hospital Federal de Bonsucesso & $6(6,1)$ \\
\hline Policlínica Piquet Carneiro & $5(5,1)$ \\
\hline HUGG & $2(2,0)$ \\
\hline C.F. Carlos Nery da Costa Filho & $1(1,0)$ \\
\hline C.F. Medalhista Olímpico Bruno Schmidt & $1(1,0)$ \\
\hline CMS Pindaro de Carvalho Rodrigues & $1(1,0)$ \\
\hline Fiocruz & $1(1,0)$ \\
\hline Hospital Federal da Lagoa & $1(1,0)$ \\
\hline Hospital Federal dos Servidores do Estado & $1(1,0)$ \\
\hline Total de Instituições Públicas & $98(100)$ \\
\hline
\end{tabular}

Fonte: Elaboração própria.

Tabela 3. Custo direto anual estimado do Sici no município do Rio de Janeiro (valores em R\$̣ Mil)

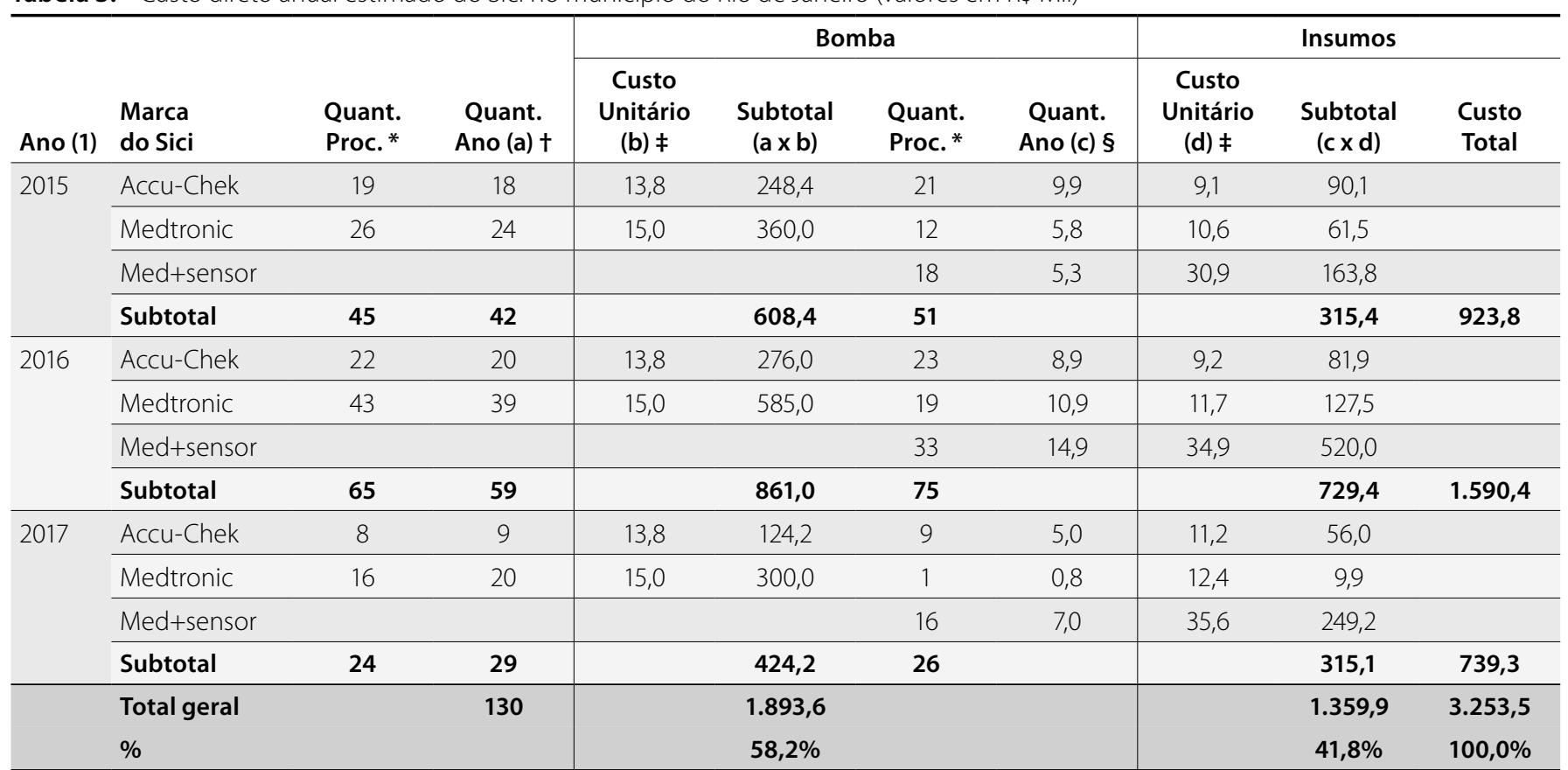

Fonte: Elaboração própria.

Notas: * Quantidade de pareceres técnicos emitidos pelo NAT, nos quais os processos judiciais foram deferidos. † A quantidade de bombas de infusão de insulina com decisões judiciais deferidas no ano (antecipação de tutela ou sentença judicial), pois o ano do parecer pode ser diferente do ano da decisão judicial. ‡ Custo unitário estimado segundo os critérios do Apêndice B. § Quantidade de insumos não padronizados para o tratamento com Sici, com decisões judiciais deferidas no ano, proporcional ao número de meses da data da decisão judicial. 
pela instituição na qual a parte autora foi atendida ou a BIC já havia sido pleiteada, por via judicial, anteriormente.

Para o cálculo do custo direto, foram considerados os processos deferidos. Como pode ser observado na Tabela 3, o custo direto estimado da judicialização dos Sici variou ano a ano, sendo $\mathrm{R} \$$ 923,8 mil em 2015, R\$ 1.590,4 mil em 2016 e R\$ 739,3 mil em 2017, acumulando um gasto total de cerca de $\mathrm{R} \$ 3,3 \mathrm{mi}$ Ihões. Desse total, 41,8\% foram relativos ao custo dos insumos e $58,2 \%$ ao custo das BIC.

Com relação ao custo por fabricante, verifica-se que 73,1\% dos gastos estão relacionados a Medtronic e 26,9\% são de gastos relativos a Accu-Chek. Tal situação decorre do fato de que a Medtronic tem um custo adicional referente à monitorização contínua de glicose intersticial e do maior número de Sici pleiteados, que foram deferidos (24 em 2015, 39 em 2016 e 20 em 2017), representando 63,8\% das 130 bombas de infusão contínua de insulina deferidas.

Vale destacar ainda que o custo da BIC da marca Medtronic é superior ao da marca Accu-Chek, sendo R\$ 15,0 mil e $\mathrm{R} \$ 13,8$ mil, respectivamente. Do mesmo modo, os custos dos insumos utilizados para o tratamento com o Sici da marca Medtronic são superiores aos da Accu-Chek.

Os custos supracitados referem-se apenas ao Sici e aos insumos que não estão padronizados, isto é, não incluem os custos do análogo de insulina, tiras reagentes, glicosímetro e lancetas.

É importante ressaltar que as quantidades indicadas pelo fabricante são, em alguns casos, diferentes das prescritas pelos médicos e, possivelmente, distintas das efetivamente utilizadas pelos pacientes. Diante do exposto, o custo para os entes decorrentes do fornecimento contínuo dos insumos deferidos também pode ser superior, uma vez que a entrega pela CADJ está vinculada à quantidade prescrita nos documentos médicos.

Embora o custo do ano de 2017 tenha diminuído em relação aos anos anteriores, assim como o número de processos deferidos, é importante esclarecer que existe um efeito cumulativo nos gastos públicos ao longo do tempo, pois os processos deferidos em anos anteriores continuam gerando custo, a cada ano, uma vez que os insumos são de uso contínuo.

O gasto anual estimado em 2017 equivale a R\$ 3,4 milhões, isto é, além do custo do próprio ano, também inclui custos dos insumos para o tratamento com Sici dos processos deferidos em 2015 e 2016, R\$ 1,0 milhão e R\$ 1,7 milhão, respectivamente (quantidade de processos deferidos multiplicado pelo custo dos insumos praticados em 2017). Consequentemente, o custo estimado acumulado no triênio alcança o montante de R\$ 6,9 milhões com judicialização do Sici.

\section{Discussão}

No que tange ao total de pareceres, observa-se um crescimento nos anos de 2013 a 2015 e uma estabilização, com pequena variação, nos anos de 2015 a 2017 que pode estar relacionada à greve dos serventuários do Tribunal de Justiça do Estado do Rio de Janeiro, iniciada em 26 de outubro de 2016 (Ordem dos Advogados do Brasil).

No ano de 2017, houve o aumento da rigidez dos critérios para emissão dos pareceres técnicos pelo NAT/TJ e NAT/JF. Dessa forma, deixaram de ser emitidos pareceres para processos nos quais os documentos médicos acostados não possuíam identificação legível do profissional emissor e para processos nos quais os documentos médicos anexados possuíssem datas de emissão com lapso temporal que pudesse interferir na inferência da necessidade dos itens pleiteados. Esse fato pode ter contribuído para a manutenção do número de pareceres.

Apesar de o Sici apresentar baixa representatividade em relação ao número total de pareceres emitidos pelo NAT/TJ e NAT/JF, nos anos de 2013 a 2017, a referida tecnologia apresenta alto custo e requer a utilização contínua de insumos não padronizados, para a realização do tratamento de uma doença que apresenta aumento de incidência mundial (International Diabetes Federation, 2017).

Durante a análise dos pareceres técnicos, foi constatada a emissão de mais de um parecer para um mesmo autor, em diversos processos, ora em decorrência da avaliação de novos documentos médicos acostados, para fornecer esclarecimento sobre algum questionamento feito no primeiro parecer, ora em decorrência da inclusão de novos pleitos no processo.

Com relação ao documento médico utilizado para dar início ao processo, no presente trabalho, foi identificado que a maior parte $(51,9 \%)$ foi emitida por profissional proveniente do SUS. Do mesmo modo, por exemplo, o estudo realizado por Marçal (2012), sobre a judicialização da Política de Assistência Farmacêutica de Pernambuco, identificou que a origem do atendimento foi a rede pública de saúde em $51,1 \%$ dos processos. Por outro lado, diversos outros estudos observaram que a origem do médico prescritor foi, na maior parte, o sistema privado (Andrade et al., 2018; Machado et al., 2011). Destaca-se ainda o exemplo do estudo de Andrade et al. (2018), que identificou que $100 \%$ dos documentos médicos utilizados para dar início ao processo judicial para a aquisição de BIC eram provenientes de médicos da rede particular de saúde.

Quanto às características relacionadas à assistência à saúde, foi observada uma concentração de prescrições por alguns médicos. Apenas nove médicos, de um total de 75 prescritores, foram responsáveis por 43,8\% das prescrições. O profissional com maior número de prescrições foi responsável por 10,1\% delas. $O$ estudo realizado por Campos et al. (2012), por exemplo, também identificou que três médicos concentraram 10,9\% das prescrições nas ações judiciais impetradas contra o estado de Minas Gerais, para a obtenção de medicamentos, entre 1999 e 2009.

Nos casos analisados, observou-se que, em 38,6\% deles (73 casos), o paciente havia sido submetido a teste com o Sici 
e que, em 39 desses casos (53,4\%), os documentos acostados eram de médicos provenientes do SUS. Tal situação chama a atenção pelo fato de que alguns médicos da rede pública estão submetendo os pacientes a tratamento com uma tecnologia não padronizada pelo SUS.

Cabe registrar que, em janeiro de 2018, a Conitec emitiu um relatório preliminar sobre o Sici no tratamento de segunda linha de pacientes com DM tipo 1. Nesse relatório preliminar, foi recomendada a não incorporação pelo SUS da referida tecnologia, pois os "membros do Plenário ponderaram que os estudos apresentados não fornecem evidências suficientes que comprovem benefícios clínicos da terapia e que a avaliação econômica é limitada e sem um modelo bem definido" (Brasil, 2018).

Neste estudo, identificou-se também que a maioria dos processos foi conduzida pela Defensoria Pública (153 casos) e que existe uma concentração de processos em um único advogado que foi responsável por 30,3\% das solicitações realizadas por advogados particulares. Marçal (2012), Barreto et al. (2013) e Santos et al. (2018) também tiveram resultados semeIhantes, identificando o Ministério Público ou Defensoria Pública como os principais responsáveis pelas solicitações.

Com relação às decisões dos juízes, o presente estudo identificou que, dos 189 processos analisados, o pleito foi deferido em 153, corroborando os estudos de Borges e Ugá (2010), que também constataram que a maioria das sentenças (89\%) foi deferida como totalmente procedentes, em primeira instância, para fornecimento de medicamentos a usuários do SUS no estado do Rio de Janeiro, em 2005.

Com relação ao custo direto da judicialização do Sici, no município do Rio de Janeiro, no período de 2015 a 2017, estima-se que esse alcance o montante acumulado de $R \$ 6,9$ milhões, no triênio, o que representa 6,7\% dos gastos com judicialização da saúde

Para apurar o custo direto governamental com a judicialização do Sici, não existe atualmente uma fonte de dados completa disponível para o acesso da população em geral. Notadamente, as análises de custos na área da saúde são indiscutivelmente importantes, pois os gestores necessitam tomar decisões sobre a alocação dos recursos disponíveis, que são escassos para atender a uma demanda tecnológica que, sabidamente, é cada vez maior.

Nesse sentido, é importante mencionar o sistema S-Codes, elaborado e implantado em 2005 pelo governo do estado de São Paulo. O referido sistema proporciona a identificação do: perfil da judicialização; custos; ranking das doenças, medicamentos, materiais, nutrição e procedimentos; ranking de médicos e serviços/profissionais de direito; identificação de duplicidade de atendimento; inativação automática de demandas sem retirada; cruzamento de dados para investigação de possíveis ilícitos na judicialização em saúde (Secretaria de Estado de Saúde, 2017).
Assim, vale destacar que o S-Codes identifica os itens adquiridos para cada processo judicial, prática que pode facilitar a mensuração dos custos diretos no estado de São Paulo.

A partir do ano de 2017, o Ministério da Saúde passou a disponibilizar sem custo, aos estados e municípios brasileiros, a plataforma web S-Codes, por meio da assinatura de um termo de cessão pelo Ministério da Saúde e pela Secretaria de Saúde de São Paulo (Brasil, 2017).

A utilização do referido sistema, no município e no estado do Rio de Janeiro, pode trazer grande benefício à gestão da judicialização da saúde, uma vez que permitirá o acesso aos gestores a informações organizadas e tempestivas relacionadas aos processos judiciais.

Outra questão que merece destaque com relação ao fornecimento dos Sici é o fato de não haver um único ente, incluindo as esferas municipal, estadual e federal, responsável pela compra e dispensação dos itens deferidos pelo Poder Judiciário, uma vez que não são padronizados pelo SUS.

Esse fato pode ocasionar uma potencial ineficiência na gestão dos gastos públicos decorrentes da judicialização dessa tecnologia, acarretando também a ausência de descontos por quantidade adquirida.

Por fim, indica-se ainda a realização de novos estudos que: (1) estendam a referida análise de custos diretos da judicialização para outras tecnologias em saúde e (2) analisem os custos diretos de todo o tratamento com Sici, incluindo os medicamentos, em todo o estado do Rio de Janeiro, objetivando estabelecer uma estimativa mais próxima do custo real da judicialização desses dispositivos.

\section{Conclusão}

O presente estudo identificou que os demandantes dos Sici por via judicial são jovens e necessitam utilizar o equipamento de forma contínua e crônica, por tempo indeterminado, podendo acarretar grande impacto para o SUS. Considerando que a tecnologia analisada é recorrentemente judicializada, os dados coletados podem gerar informações úteis para os gestores governamentais da área da saúde, na avaliação da eficiência na alocação dos recursos públicos, para os profissionais que trabalham na assistência judiciária e para os juízes.

\section{Referências bibliográficas}

Accu-Chek Combo [homepage]. Guia Rápido de Manuseio. Available from: https:// debemcomavida.accuchek.com.br/media/354089/guia-rapido-manuseiosanfona_juntos-conectados__hm_.pdf. Accessed on: Nov 12, 2018.

Andrade RSG, Santos ECB, Teixeira CRS, Batista JMF, Arrelias CCA, Otero LM, et al. Processos judiciais para aquisição de bomba de insulina em Ribeirão Preto. Rev Bioét. 2018 [citado 2018 Dez 20];26(1). Available from: http://revistabioetica.cfm. org.br/index.php/revista_bioetica/article/view/1478/1807.

Barreto JL, Pereira MT, Guimarães MCL, Penaforte TR, Formigli VLA. Perfil das demandas judiciais por medicamentos em municípios do Estado da Bahia. Revista Baiana de Saúde Pública. 2013[citado 2018 Out 22];37(3). Available from: http://repositorio.ufba.br:8080/ri/handle/ri/21954. 
Boing A, Bloemer NS, Roesler C, Fernandes S. A judicialização do acesso aos medicamentos em Santa Catarina: um desafio para a gestão do sistema de saúde. Revista de Direito Sanitário. 2013[citado 2017 Nov 10];14(1). Available from: http://www.revistas.usp.br/rdisan/article/view/56625.

Borges DCL, Ugá MAD. Conflitos e impasses da judicialização na obtenção de medicamentos: as decisões de 1a instância nas ações individuais contra o Estado do Rio de Janeiro, Brasil, em 2005. Cad Saúde Pública. 2010;26(1):59-69.

Brasil. Ministério da Saúde. Avaliação de Tecnologias em Saúde Ferramentas para a Gestão do SUS. Brasília, DF: 2009. Available from: http://bvsms.saude.gov.br/bvs/ publicacoes/avaliacao_tecnologias_saude_ferramentas_gestao.pdf. Accessed on: Jan 4, 2019].

Brasil. Ministério da Saúde. Comissão Nacional de Incorporação de Tecnologias no SUS. Bomba de infusão de insulina como adjuvante no tratamento de segunda linha de pacientes com diabetes mellitus tipo 1. Relatório de Recomendação nº 375. Brasília, DF: Ministério da Saúde; 2018.

Brasil. Ministério da Saúde. Comissão Nacional de Incorporação de Tecnologias no SUS. Insulinas análogas de ação rápida para diabetes mellitus tipo 1. Relatório de Recomendação n² 245. Brasília, DF: Editora MS; 2017.

Brasil. Ministério da Saúde. Decreto no 7.646, de 21 de dezembro de 2011. Dispõe sobre a Comissão Nacional de Incorporação de Tecnologias no Sistema Único de Saúde e sobre o processo administrativo para incorporação, exclusão e alteração de tecnologias em saúde pelo Sistema Único de Saúde - SUS, e dá outras providências. Diário Oficial da União 22 dez. 2011.

Brasil. Ministério da Saúde [homepage]. S-Code. Available from: http://portalms. saude.gov.br/artigos/41922-scode. Accessed on: Aug 27, 2018.

Brasil. Ministério da Saúde. Política Nacional de Gestão de Tecnologias em Saúde. Brasilia, DF: 2010. Available from: http://bvsms.saude.gov.br/bvs/publicacoes/ politica_nacional_gestao_tecnologias_saude.pdf. Accessed on: Jan 5, 2019.

Brasil. Ministério da Saúde. Portaria no 11, de 13 de março de 2017. Torna pública a decisão de incorporar caneta para injeção de insulina humana NPH e insulina humana regular no âmbito do Sistema Único de Saúde - SUS. Diário Oficial da União 14 mar. 2017.

Brasil. Ministério da Saúde. Relação Nacional de Medicamentos Essenciais. Brasília, DF: Editora MS; 2017.

Brasil. Ministério da Saúde. Secretaria de Atenção à Saúde. Portaria Conjunta no 8, de 15 de março de 2018. Aprova o Protocolo Clínico e Diretrizes Terapêuticas do Diabete Melito Tipo 1. 2018. Available from: http://portalarquivos2.saude.gov. br/images/pdf/2018/marco/19/Po/rtaria-Conjunta-n-8.pdf. Accessed on: Aug 18, 2018.

Campos NOH, Acurcio FA, Machado MAA, Ferré F, Barbosa FLV, Cherchiglia ML. Médicos, advogados e indústria farmacêutica na judicialização da saúde em Minas Gerais, Brasil. Rev Saúde Pública. 2012[citado 2017 Set 3];46(5). Available from: http://www.scielo.br/scielo.php?script=sci_arttext\&pid=S0034$89102012000500004 \& \operatorname{lng}=e n$.

Diretoria Geral de Gestão do Conhecimento (DGCON). Biblioteca do Tribunal de Justiça do Estado do Rio de Janeiro. Ato Normativo TJ no 5, de 2012. Available from: http://www.jir.jus.br/documents/10136/1217804/01-02-2012.pdf. Accessed on: May 10, 2018.

Fatourechi MM, Kudva YC, Murad MH, Elamin MB, Tabini CC, Montori VM. Clinical review: Hypoglycemia with Intensive Insulin Therapy: A Systematic Review and Meta-Analyses of Randomized Trials of Continuous Subcutaneous Insulin Infusion Versus Multiple Daily Injections. J Clin Endocrinol Metab. 2009;94(3):729-40.

Ferreira SL, Costa AM. Núcleos de Assessoria Técnica e Judicialização da Saúde: constitucionais ou inconstitucionais? Rev SJRJ. 2013[citado 2017 Abr 15];20(36). Available from: http://www.uff.br/siddharta_legale/files/2014/07/Nucleos-deassessoria-tecnica-e-judicializa\%C3\%A7\%C3\%A30-da-sa\%C3\%BAde.pdf.

Figueiredo TA. Análise dos medicamentos fornecidos por mandado judicial na Comarca do Rio de Janeiro: a aplicação de evidências científicas no processo de tomada de decisão [dissertação]. Rio de Janeiro: Escola Nacional de Saúde Pública Sérgio Arouc; 2010.
Guimarães RCMG, Palheiro PHD. Medidas adotadas para enfrentar a judicialização na Secretaria de Saúde do Estado do Rio de Janeiro e a experiência da Câmara de Resolução de Litígios de Saúde. Rev Saúde Pública. 2011 [citado 2017 Maio 13];45(3). Available from: http://www.conass.org.br/biblioteca/pdf/colecao2015/ CONASS-DIREITO_A_SAUDE-ART_33.pdf.

International Diabetes Federation. Atlas de la Diabetes de la FID. 8 a ed. Genebra: International Diabetes Federation; 2017.

Justiça Federal. Seção Judiciária do Rio de Janeiro. Processo JFRJ-ADM-2015/00040 Acordo de Cooperação Secretaria de Saúde. Available from: https://www.jfrj.jus. br/sites/default/files/SEGAJ/acordo_nat_2015.pdf. Accessed on: Jun 18, 2017.

Machado MAA, Acurcio FA, Brandão CMR, Faleiros DR, Guerra JAA, Cherchiglia ML, et al. Judicialização do acesso a medicamentos no Estado de Minas Gerais, Brasil. Rev Saúde Pública. 2011[citado 2018 Jun 22];45(3). Available from: http://www. scielo.br/scielo.php?script=sci_arttext\&pid=\$0034-89102011000300018\&lng=en.

Marçal KKS. A judicialização da assistência farmacêutica: o caso Pernambuco em 2009 e 2010 [dissertação]. Recife: Fundação Oswaldo Cruz; 2012.

Messeder AM, Osorio-de Castro CGS, Luiza VL. Mandados judiciais como ferramenta para garantia do acesso a medicamentos no setor público: a experiência do Estado do Rio de Janeiro. Cad Saúde Pública. 2005;21(2):525-34.

National Institute for Health and Care Excellence - NICE. Continuous subcutaneous insulin infusion for the treatment of diabetes mellitus. Technology appraisal guidance [TA151]. Published date: 23 July 2008. Reviewed in: May 2011. Available from: https://www.nice.org.uk/guidance/ta15.

Oliveira MRM, Delduque MC, Sousa MF, Mendonça AVM. Judicialização da saúde: para onde caminham as produções científicas? Rev Saúde Debate. 2015[citado 2018 Out 10];105(39). Available from: http://www.scielo.br/scielo.php?script=sci_ arttext\&pid=S0103-11042015000200525\&lng=en\&nrm=iso.

Ordem dos Advogados do Brasil. Serventuários do TJ/RJ entram em greve por tempo indeterminado - TJ corta o ponto [homepage]. 8a Subseção São Gonçalo. Available from: http://www.oabsg.com.br/2016/10/27/serventuarios-do-tjijentram-em-greve-por-tempo-indeterminado/. Accessed on: Jan 15, 2019.

Paim LFNA, Batt CR, Saccani G, Guerreiro ICK. Qual é o custo da prescrição pelo nome de marca na judicialização do acesso aos medicamentos? Cad Saúde Colet. 2017;25(2):201-9.

Pierro B. Demandas crescentes. Revista Pesquisa FAPESP. 2017[citado 2017 Maio 5];252. Available from: https://revistapesquisa.fapesp.br/2017/02/10/demandascrescentes/.

Poder Judiciário do Estado do Rio de Janeiro. Núcleo de Assessoria Técnica é considerado referência nacional por secretários estaduais e municipais do país. Available from: http://www.jir.jus.br/noticias/noticia/-/visualizarconteudo/5111210/5162771. Accessed on: Jun 25, 2017.

Santos ECB, Teixeira CRS, Zanetti ML, Istilli PT, Pereira LHTR, Torquato MTCG. Judicialização da saúde: acesso ao tratamento de usuários com diabetes mellitus. Texto Contexto Enferm. 2018[citado 2018 Nov 25];27(1). Available from: http://www.scielo.br/scielo.php?script=sci_arttext\&pid=S010407072018000100321\&lng=en.

Secretaria de Estado de Saúde de São Paulo [homepage]. Sistema S-CODES Available from: http://portalarquivos2.saude.gov.br/images/pdf/2017/ julho/28/1-a-APRESENTACAO-S-CODES-PARA-CIT.pdf. Accessed on: Nov 13, 2018.

Silva MV. O processo decisório judicial e a assessoria técnica: a argumentação jurídica e médico-sanitária na garantia do direito à assistência terapêutica no Sistema Único de Saúde [tese]. Rio de Janeiro: Fundação Oswaldo Cruz; 2012.

Sociedade Brasileira de Diabetes. Diretrizes da Sociedade Brasileira de Diabetes 20172018. São Paulo: Clamando Editora Científica; 2017. 


\section{APÊNDICE A - CUSTO DOS INSUMOS NÃO PADRONIZADOS}

\begin{tabular}{|c|c|c|c|c|c|c|c|}
\hline Insumos Não Padronizados & $\begin{array}{c}\text { Quantidade } \\
\text { Anual * }\end{array}$ & \multicolumn{2}{|c|}{2015} & \multicolumn{2}{|c|}{2016} & \multicolumn{2}{|c|}{2017} \\
\hline Conjunto de infusão & 122 & 60,40 & $7.368,80$ & 60,40 & $7.368,80$ & 73,15 & $8.924,30$ \\
\hline Aplicador de conjunto de infusão & 1 & 190,00 & 190,00 & 190,00 & 190,00 & 271,70 & 271,70 \\
\hline \multirow[t]{2}{*}{ Pacote de serviços } & 3 & 167,74 & 503,22 & 185,00 & 555,00 & 203,50 & 610,50 \\
\hline & & & $9.074,62$ & & $9.241,08$ & & $11.216,82$ \\
\hline \multicolumn{8}{|l|}{ Painel 2 - itens do Sici Medtronic } \\
\hline Conjunto de infusão (MMT-396 ou 397) & 122 & 69,50 & $8.479,00$ & 76,50 & $9.333,00$ & 81,30 & $9.918,60$ \\
\hline CareLink USB (MMT-7305NA) & & & $10.615,00$ & & $11.688,80$ & & $12.384,20$ \\
\hline \multicolumn{8}{|c|}{ Painel 3 - itens da Monitorização Contínua da Glicose - Medtronic } \\
\hline Sensores de glicose (MMT-7708A) & 61 & 292,00 & $17.812,00$ & 335,80 & $20.483,80$ & 335,80 & $20.483,80$ \\
\hline Aplicador do sensor (MMT-7510) & 1 & 150,00 & 150,00 & 165,00 & 165,00 & 165,00 & 165,00 \\
\hline \multirow[t]{2}{*}{ Transmissor MiniLink (MMT-7707NA) } & 1 & $2.343,00$ & $2.343,00$ & $2.577,00$ & $2.577,00$ & $2.577,00$ & $2.577,00$ \\
\hline & & & $20.305,00$ & & $23.225,80$ & & $23.225,80$ \\
\hline
\end{tabular}

Fonte: Elaboração própria.

(Valores em R\$)

Nota: *Quantidades-padrão estimadas conforme indicado no Apêndice B. † Preços apurados com base (a) em informações de compra (custo incorrido na aquisição ou custo real) de 2015 disponibilizadas, em 09/05/2018, pela CADJ (Central de Atendimento de Demandas Judiciais) da Secretaria Estadual de Saúde do Rio de Janeiro ou (b) orçamento mais conservador disponibilizado pelos fornecedores (fabricante/distribuidor). ‡ Na ausência de informações sobre o preço, foi considerado o valor do ano posterior ou anterior disponível.

\section{APÊNDICE B - LISTA DOS INSUMOS NÃO PADRONIZADOS}

\begin{tabular}{|c|c|c|c|c|c|}
\hline Insumos Não Padronizados & Descrição de Uso & & \multicolumn{3}{|c|}{ Quantidade Anual } \\
\hline \multicolumn{6}{|l|}{ Painel 1 - itens do Sici Accu-Chek } \\
\hline Conjunto de infusão & & (cx. 10) & & & \\
\hline - Cânula & Deve ser trocada a cada 2 ou 3 dias $+\S$ & & 122 & 122 & 183 \\
\hline Aplicador de conjunto de infusão & Item único & & --- & 1 & --- \\
\hline Reservatório & Deve ser usado por, no máximo, 6 dias † $\S$ & (cx. 25) & 61 & 61 & --- \\
\hline \multicolumn{6}{|l|}{ Pacote de Serviços } \\
\hline - Pilha (bateria) & Deve ser trocada a cada 4 meses $\|$ & & --- & 3 & --- \\
\hline Aplicador de conjunto de infusão & Uma unidade permanente $\|$ & & --- & 1 & --- \\
\hline Reservatório & Deve ser trocado a cada 3 dias $\|$ & $(c x .10)$ & --- & 122 & --- \\
\hline
\end{tabular}




\begin{tabular}{|c|c|c|c|c|}
\hline A cada 6 dias, no máximo * $\S$ & $(c x .5)$ & 61 & 61 & --- \\
\hline |tem único || & & --- & 1 & --- \\
\hline |tem único || & & --- & 1 & --- \\
\hline
\end{tabular}

Fonte: Elaboração própria.

Nota: Informações de uso (quantidade) estão descritas * nos Manuais dos fabricantes do Sici; † no Guia Rápido de Manuseio Accu-Chek Combo; ‡ no Tutorial de uso Medtronic Paradigma Veo; § nas Diretrizes da SBD 2017-2018 - Sociedade Brasileira de Diabetes (http://www.diabetes.org.br/ebook/component/k2/item/58-o-papeldas-bombas-de-insulina-na-estrategia-de-tratamento-do-diabetes-tipo-1-dm1); e || nos orçamentos fornecidos pelos fabricantes/distribuidores, contidos nos processos analisados. Não Informado - informação não disponível.

\section{APÊNDICE C - GASTOS NO ESTADO DO RIO DE JANEIRO EM PROCESSOS JUDICIAIS NA ÁREA DA SAÚDE}

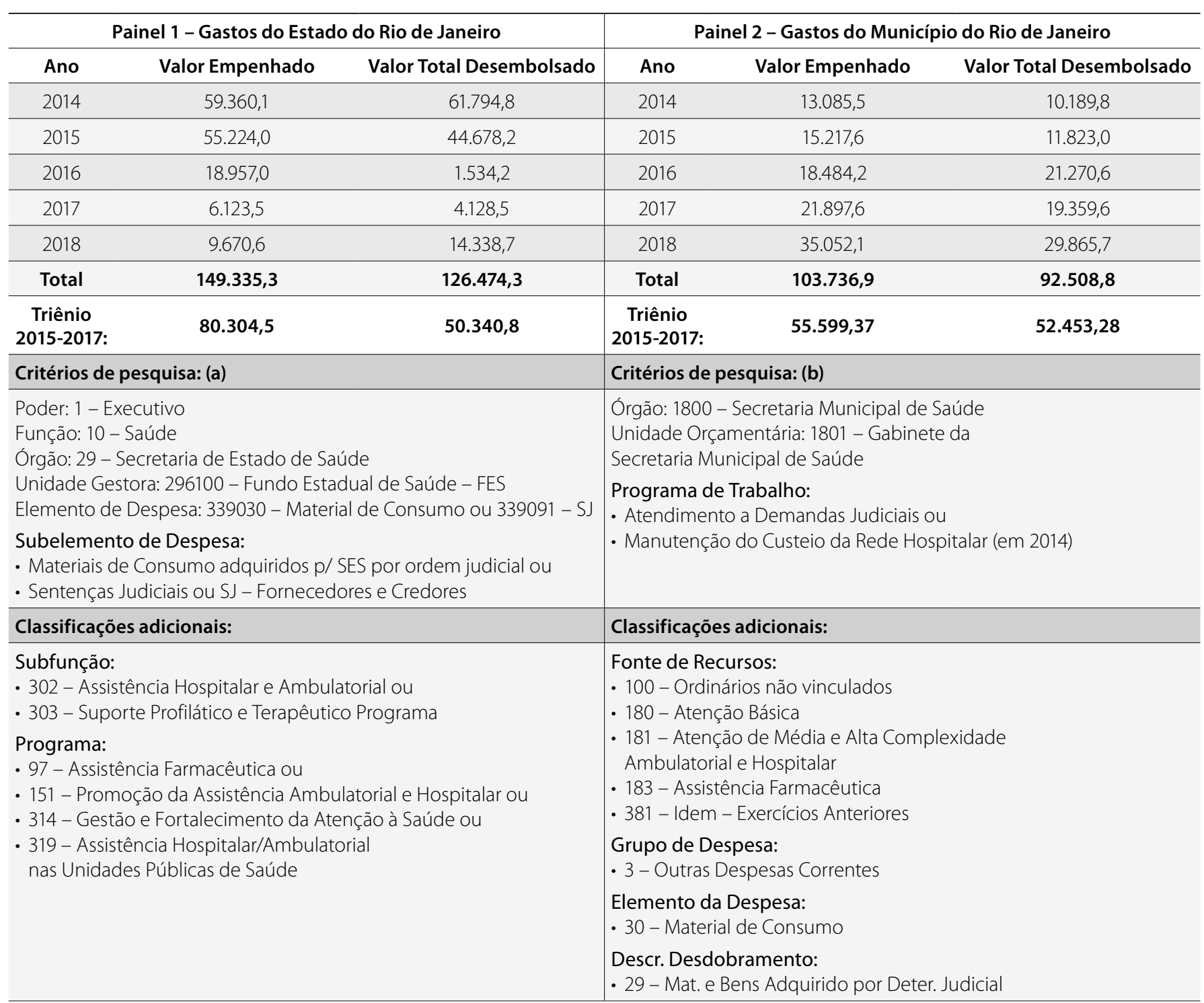

Fonte: Elaboração própria.

Notas: Dados disponíveis no (a) Portal de Transparência do Governo do Estado do Rio de Janeiro (http://www.transparencia.rj.gov.br/) [opção: "Despesas" e "Consulta livre"]; ou (b) Portal de Transparência do Município do Rio de Janeiro - Rio Transparente (http://riotransparente.rio.rj.gov.br) [opção: "Despesas" e "Por Órgão"], pesquisa em 7 de janeiro de 2019. (c) Os valores indicados na tabela não incluem os sequestros de verbas (depósitos judiciais) ocorridos para aquisição da tecnologia por parte do paciente. (d) SJ - Sentenças Judiciais. 